\title{
Atrioventricular and interventricular delay optimization in cardiac resynchronization therapy: physiological principles and overview of available methods
}

\author{
Patrick Houthuizen • Frank A. L. E. Bracke • \\ Berry M. van Gelder
}

Published online: 14 December 2010

(C) The Author(s) 2010. This article is published with open access at Springerlink.com

\begin{abstract}
In this review, the physiological rationale for atrioventricular and interventricular delay optimization of cardiac resynchronization therapy is discussed including the influence of exercise and long-term cardiac resynchronization therapy. The broad spectrum of both invasive and non-invasive optimization methods is reviewed with critical appraisal of the literature. Although the spectrum of both invasive and non-invasive optimization methods is broad, no single method can be recommend for standard practice as large-scale studies using hard endpoints are lacking. Current efforts mainly investigate optimization during resting conditions; however, there is a need to develop automated algorithms to implement dynamic optimization in order to adapt to physiological alterations during exercise and after anatomical remodeling.
\end{abstract}

Keywords Cardiac resynchronization therapy · Heart failure - Physiology · Atrioventricular delay · Interventricular delay

\section{Introduction}

In patients with symptomatic systolic heart failure and prolonged QRS duration, cardiac resynchronization therapy (CRT) has proven to be of additional value on top of recommended medical therapy $[1,2]$. Nevertheless, there remain a considerable number of non-responders to CRT that can be as high as 30\% [3]. The non-response can be partly

P. Houthuizen $(\varangle)$ · F. A. L. E. Bracke - B. M. van Gelder Department of Cardiology, Catharina Hospital, P.O. Box 1350, 5602 ZA Eindhoven, The Netherlands

e-mail: cardiology@houthuizen.be caused by inappropriate settings of atrioventricular (AV) and interventricular (VV) intervals leading to persistent atrioventricular, interventricular and intraventricular dyssynchrony. In this review, we will discuss the physiological and pathophysiological rationale for AV and VV optimization followed by an overview of available optimization methods.

\section{Physiological electrical activation and mechanical contraction}

A coordinate contraction sequence of the heart chambers is facilitated by rapid activation via the specialized conduction system. The cardiac action potential originates in the sinus node and reaches the atrioventricular node (AV node) within 100 milliseconds (ms). Slowing of conducting through the AV node delays the onset of ventricular activation with approximately $80 \mathrm{~ms}$ to allow optimal atrial contribution to ventricular preload. Rapid conduction of the electrical impulse through the His bundle, bundle branches and the Purkinje system activates the whole left ventricle (LV) within 60-80 ms. Ventricular activation proceeds from subendocardially located breakthroughs of the bundle branches to the epicardium in a centrifugally and tangentially direction [4].

Cardiac output is dependent on preload (Frank-Starling relation), afterload and myocardial contractility. The latter is not only influenced by neurohormones, but also dependent on heart rate (staircase phenomenon or Bowditch effect) and afterload (Anrep effect). Autonomic and neurohormonal regulatory mechanisms ensure adequate cardiac output under varying physiological conditions. Regulation and feedback is provided by pressure sensors in the venous and arterial vascular system [5]. 
Sympathetic stimulation at increasing heart rate shortens AV delay and ventricular systole, thus preventing atrial systole to occur against a closed mitral valve during exercise. Shortening of ventricular systole also enables longer ventricular filling time [6].

\section{Pathophysiological electrical activation and mechanical contraction}

Apart from decreased myocardial contractility, there are several other causes for decreased cardiac output in heart failure. First, in a subset of patients, there is a disturbance in coordination of atrial and ventricular activation with suboptimal timing of atrial contraction ( $A V$ dyssynchrony).

The atrial contraction enhances ventricular preload by optimizing sarcomere length of ventricular myocytes prior to contraction which in turn increases LV stroke volume. This booster function generates an increase in LV enddiastolic pressure at a relatively low mean venous pressure, thus protecting the pulmonary system from edema [7]. In case of a shortened or prolonged AV conduction, this preload enhancement is diminished or even lost. As the atrial booster effect also contributes to timely closure of the atrioventricular valves, a prolonged AV delay can also lead to premature inversion of the atrioventricular pressure gradient resulting in diastolic mitral regurgitation $[6,8]$.

Secondly, a large number of heart failure patients have ventricular conduction disturbances, predominantly left bundle branch block (LBBB). Although the term "block" suggests an abrupt interruption of conduction, there is a spectrum of conduction abnormalities varying from a proximal barrier to a more diffuse slowing of conduction. As a consequence, the LV is electrically activated throughout myocardial tissue [9]. Compared to the specialized conduction system, conduction velocity in myocardial tissue is slower and the activation front spreads preferably in a circumferential than in a perpendicular direction [10]. This can lead to mechanical interventricular (and intraventricular) dyssynchrony.

Because of their serial alignment and intimate anatomical relationship, the mechanical properties of both ventricles are influenced by each other. This close interaction is further influenced by the interventricular septum and pericardium. Changes in preload or afterload of one ventricle alter the pressure in the other ventricle [11, 12]. Although this interaction is negligible in the healthy heart, both systolic and diastolic interactions are augmented in case of heart failure [13, 14]. Difference in activation timing with the right ventricle $(\mathrm{RV})$ contracting earlier than the LV (as with LBBB) deteriorates LV function $[15,16]$.

Thirdly, asynchronous electrical activation of the LV in case of LBBB leads to an altered contraction pattern.
Initially, the septum shortens during the isovolumic contraction time, causing an early systolic stretching of the opposing, still non-activated posterolateral wall. Eventually, this posterolateral wall is activated late and exhibits a late systolic or even post-systolic shortening after the aforementioned early systolic stretching. This intraventricular dyssynchrony reduces the efficiency of the LV pumping function as part of the metabolic energy is wasted in intraventricular volume shifts rather than in ventricular ejection [17].

\section{Atrioventricular and interventricular synchronization in CRT}

Physiological rationale for optimization

As outlined above, from a physiological point of view, it seems reasonable to assume that correction of atrio-, interand intraventricular dyssynchrony improves cardiac function and efficiency. In the contemporary era of CRT, this can be achieved by programming both AV and VV timings.

It should be stressed that intrinsic $\mathrm{AV}$, programmed $\mathrm{AV}$ and programmed VV delay can all influence ventricular activation and filling. Thus, depending on the device settings, there can be up to three activation fronts that potentially determine the degree of intraventricular dyssynchrony: intrinsic right bundle branch activation, right and left ventricular pacing, respectively (Fig. 1) [16].

The interaction between these three activation fronts is illustrated in Fig. 2 showing a 12-lead electrocardiogram (ECG) recording during three different programmed AV delays in two patients with a CRT device. In Fig. 2a, it can
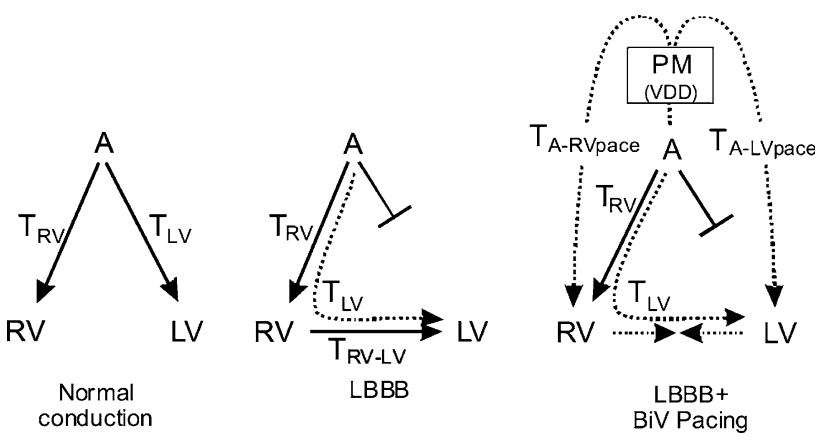

Fig. 1 Schematic pathway of different ventricular activation fronts during normal conduction, LBBB and LBBB with biventricular pacing. During normal conduction (left), activation of right ventricle (RV) and left ventricle (LV) occurs through intrinsic activation, and the time of activation $\left(T_{\mathrm{RV}}\right.$ and $\left.T_{\mathrm{LV}}\right)$ is similar. During LBBB (middle), activation to the $\mathrm{LV}$ lateral wall $\left(T_{\mathrm{LV}}\right)$ is delayed because of slow myocardial conduction $\left(T_{\mathrm{RV}-\mathrm{LV}}\right)$. During biventricular pacing (right) $\mathrm{RV}$ and $\mathrm{LV}$ lateral wall can be activated by a pacing stimulus ( $T_{\mathrm{A}-\mathrm{RV} \text { pace }}$ and $T_{\mathrm{A}-\mathrm{LV} \text { pace, }}$, respectively) if stimulation occurs before intrinsic activation. (From Vernooy et al. [16], with permission) 

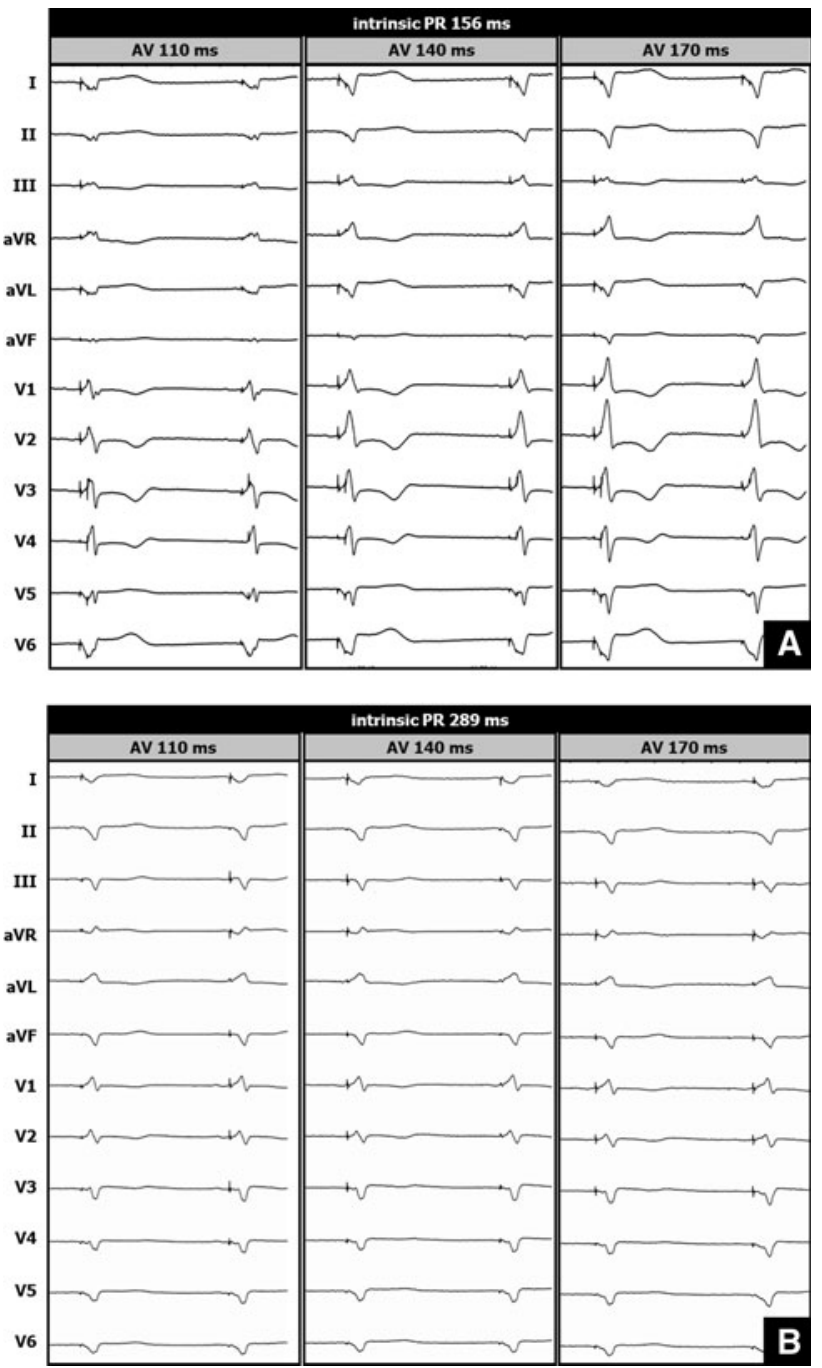

Fig. 2 Registration of 12-lead electrocardiogram in two patients with different intrinsic conduction during biventricular pacing with varying AV delays. Panel $A$ in this patient with an intrinsic PR interval of $156 \mathrm{~ms}$, there is progressive change in QRS morphology between different AV delays. At an AV delay of $170 \mathrm{~ms}$, there is maximal contribution of all 3 activation fronts resulting in the smallest QRS complex. Panel $B$ in case of a very long intrinsic PR interval of $289 \mathrm{~ms}$, there is no change in QRS complex at different AV delays as intrinsic conduction does not contribute to ventricular activation

be appreciated that there is a progressive change in QRS morphology between the AV delays. As the intrinsic PR interval of this patient is $156 \mathrm{~ms}$, the smallest QRS complex is seen at a programmed AV delay of $170 \mathrm{~ms}$ allowing maximal contribution of all three activation fronts. In Fig. 2b on contrary, no change in QRS morphology is noted between the AV delays. The intrinsic PR interval of this patient is $289 \mathrm{~ms}$, and therefore, intrinsic conduction does not contribute to ventricular activation.

Most patients not only have variable intra-atrial, interventricular and intraventricular conduction delays, but also different positions of right atrial, RV and LV leads, making it difficult to predict the optimal AV and VV timings [18]. This supports the concept of an individualized and tailored optimization of AV and VV timings.

The importance of AV and VV optimization has already been shown in general pacing. RV single chamber pacing disturbs the temporal relation between atria and ventricles leading to decreased ventricular performance especially in case of compromised cardiac function [19, 20]. In the early nineties, the use of DDD pacing was proposed in patients with refractory terminal heart failure and a long atrioventricular delay [21]. It was anticipated that improvement in the atrioventricular dyssynchrony by sequential atrioventricular pacing would lead to improved outcome. However, this potentially beneficial effect was hampered by the aggravated inter- and intraventricular dyssynchrony caused by RV pacing [22]. These observations have set the base for the current therapy of biventricular pacing.

It has been demonstrated that diastolic mitral valve regurgitation (MR) can be reversed by $\mathrm{AV}$ sequential pacing with short AV intervals [23]. The mechanism for improvement in functional systolic MR is more complex. It is caused by an imbalance between closing and tethering forces on the mitral valve leaflets. Due to LV and mitral valve annular dilation, there is a restrictive leaflet motion requiring a higher (systolic) transmitral pressure gradient to close the valve [24]. Moreover, LV dyssynchrony can lead to dyscoordinate contraction of both papillary muscles contributing to a synchronization of tethering forces [25]. In contrast, closing forces are reduced as a consequence of decreased LV systolic function. CRT improves LV systolic function and can result in an immediate reduction of MR [24]. In patients with late activation of the posterior papillary muscle, an acute reduction can also be observed with CRT. Long-term resynchronization induces LV reverse remodeling with reduction in $\mathrm{LV}$ and mitral annular dimension resulting in further improvement in MR [26].

\section{Evidence for atrioventricular optimization}

The beneficial effect of optimizing AV timing has been mainly investigated in patients with an indication for permanent dual-chamber (right atrial and RV) pacing. The majority of these small-scale, non-randomized studies focus on acute hemodynamic effects of atrioventricular optimization without evaluation of long-term morbidity and mortality. However, these results cannot be directly extrapolated to the CRT population [27].

In a CRT population, the PAcing THerapies in Congestive Heart Failure (PATH-CHF) trial demonstrated a significant acute hemodynamic effect of varying the AV delay in RV, LV and biventricular pacing [28, 29]. Interestingly, the optimal AV delay for left ventricular $\mathrm{d} P / \mathrm{d} t_{\max }$ (LV $\mathrm{d} P / \mathrm{d} t_{\max }$ ) was significantly shorter for $\mathrm{RV}$ and 
Fig. 3 During LV pacing, there is fusion at $\mathrm{AV}$ interval 1 (AV1); however, no fusion is observed at shorter AV intervals (AV2, AV3 and AV4), as can be appreciated from the 12-lead electrocardiogram. Notice the change in morphology of the RV electrogram (RV EGM) when there is no fusion with the intrinsic RBB. (From van Gelder et al. [31], with permission)
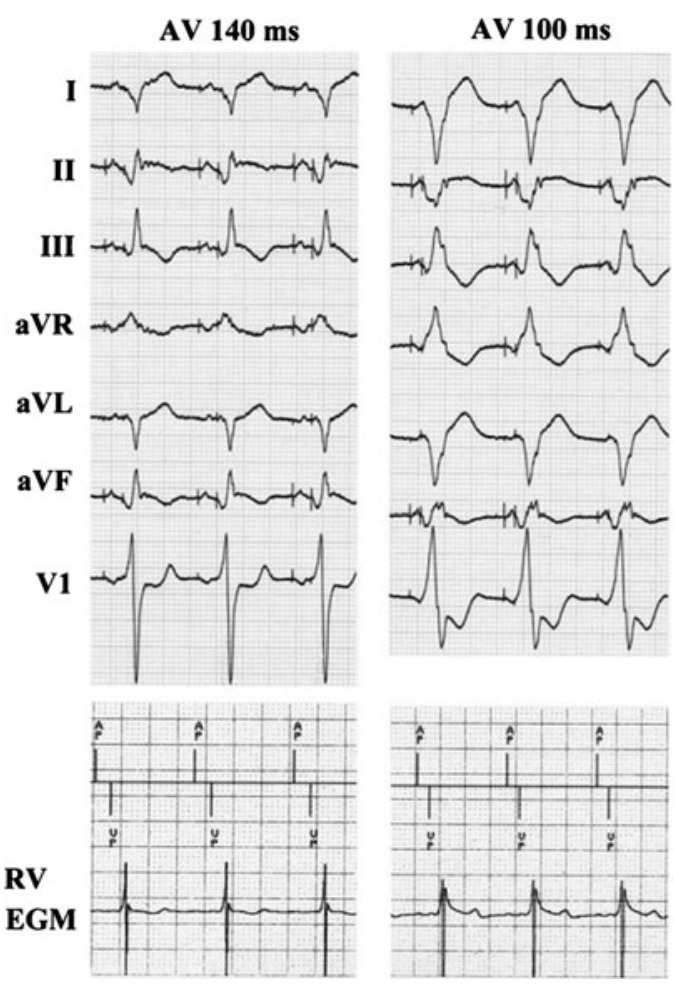
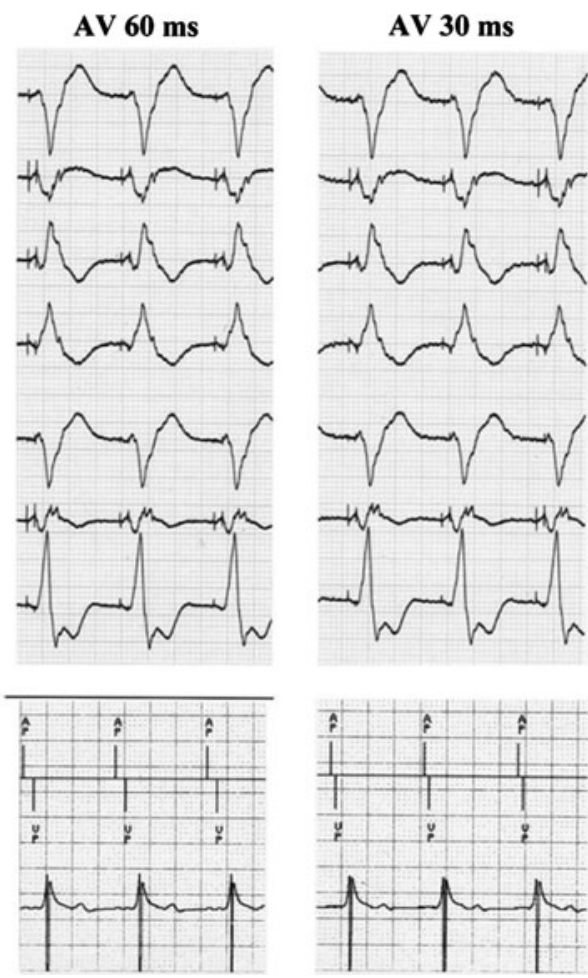

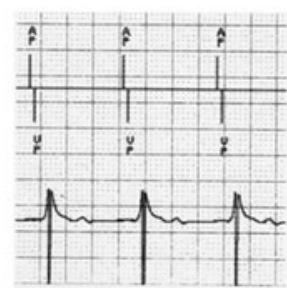

biventricular pacing compared to LV pacing in the group of responders. This variable acute hemodynamic response to different AV delays was also observed in the PATH-CHFII trial [30]. This could be explained by the fact that during left ventricular pacing, a left-sided atrioventricular delay is set which should be longer to allow fusion with intrinsic conduction coming from the normal-conducting right bundle branch (Fig. 1).

An example of the effect of varying AV delay during left ventricular pacing is shown in Fig. 3. At an AV delay that is programmed $40 \mathrm{~ms}$ shorter than the intrinsic PR interval (indicated as "AV1" in Fig. 3), there is fusion with intrinsic right bundle branch conduction which can be appreciated from the surface ECG and RV electrogram. Fusion is lost with shorter AV delays (AV2, AV3 and AV4) [31].

Atrial sensing or atrial pacing will result in different optimal AV delays and has to be accounted for during optimization. Compared to atrial sensing, the optimal AV delay needs to be prolonged during atrial pacing in order to obtain similar synchronization. In practice, one could first optimize AV and VV delays during atrial pacing. To get the same resynchronization, it suffices to adjust the AV delay during atrial sensing to match QRS morphology of the optimal AV delay obtained during atrial pacing (Fig. 4) [32].

Only a small number of prospective and/or randomized clinical studies compare the optimization of AV delay to an empirical AV delay. Although these studies are small scale and use different optimization techniques, optimization of the AV delay shows a significant beneficial effect on acute hemodynamic response, New York Heart Association (NYHA) class, LV ejection fraction and brain natriuretic peptide level [33-35].

So far, there has been no large-scale, prospective and randomized trial evaluating the effect of $\mathrm{AV}$ optimization on morbidity and mortality. Nevertheless, most large CRT trials applied some form of AV delay optimization [1-3, 18]. It is unknown whether the beneficial effects of CRT in these trials would also be present without AV delay optimization. Based on the trials' methodology and results, current guidelines of the European Society of Cardiology are recommended to optimize the AV delay [36].

Evidence for interventricular optimization

The relative position of right and left ventricular leads also influences timing of activation. As a consequence, VV optimization may compensate for suboptimal lead placement [37]. However, even in case of optimal lead placement, VV delay optimization can be of importance: some patients exhibit a significant delay between LV pacemaker stimulation and LV depolarization which can be counteracted by pre-exciting the $\mathrm{LV}$ pacing lead relative to the RV pacing lead (Fig. 5).

None of the larger CRT trials included VV optimization in their protocol, partly because this feature was not available at time of inclusion [38]. In smaller studies, an 
Fig. 4 Example of the difference in optimal paced AV (PAV) interval and optimal sensed AV (SAV) interval. In this patient, CRT was optimized during sequential $\mathrm{AV}$ pacing using $\mathrm{LV} \mathrm{d} P / \mathrm{d} t_{\max }$. The optimal PAV interval was $150 \mathrm{~ms}$ resulting in a $\mathrm{LV} \mathrm{d} P / \mathrm{d} t_{\max }$ of $862 \mathrm{mmHg} / \mathrm{s}$. To determine the optimal SAV interval, the stimulation rate was reduced below the intrinsic sinus rate, and a 12-lead electrocardiogram was recorded during incremental shortening of the SAV interval. At a SAV interval of $110 \mathrm{~ms}$, the QRS complex matches the QRS complex at the optimal PAV interval.

Determination of optimal SAV interval is also confirmed by the $\mathrm{LV} \mathrm{d} P / \mathrm{d} t_{\max }$ measurement
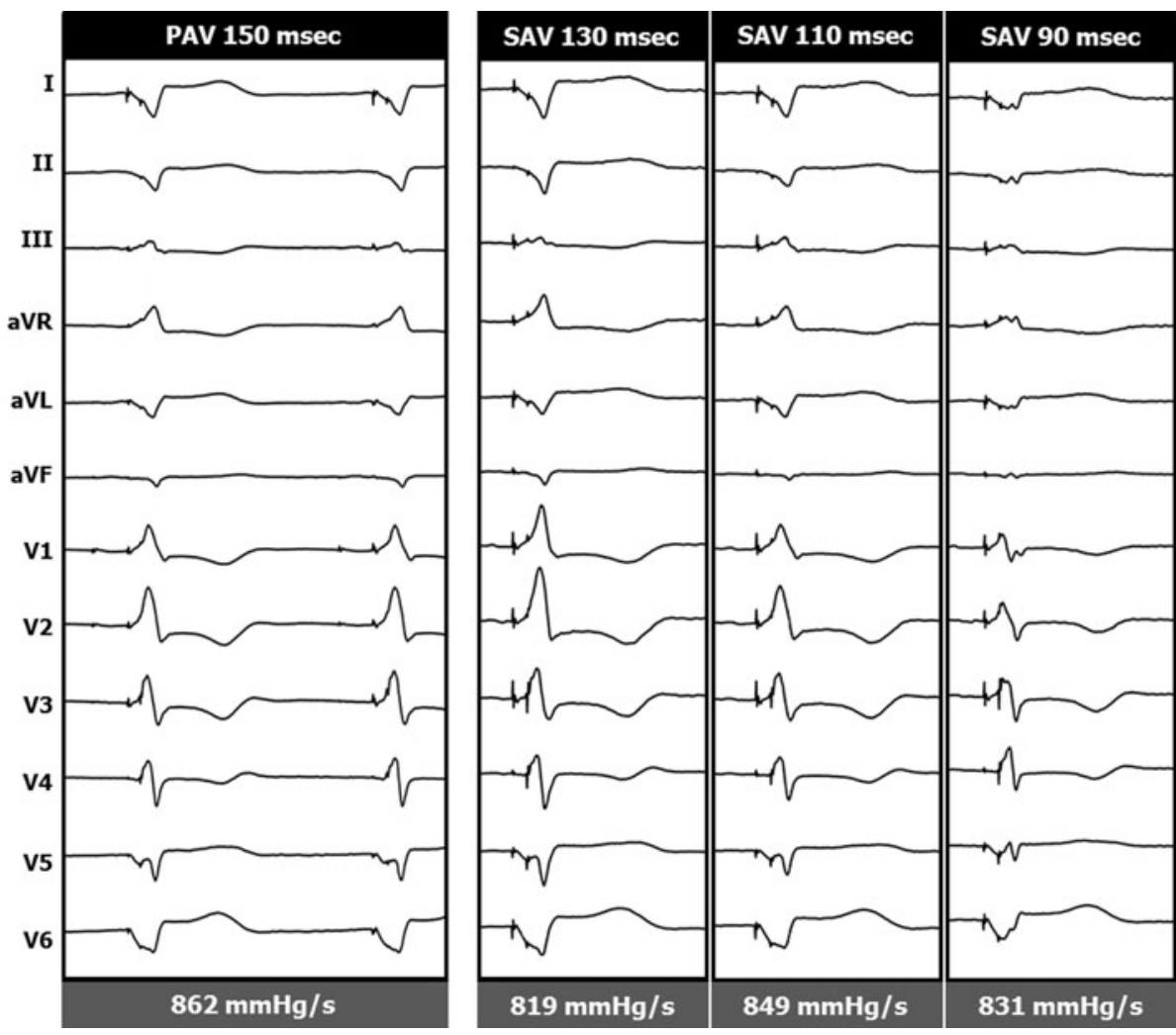

Fig. 5 Twelve-lead electrocardiogram recording during RV pacing (left panel), $\mathrm{LV}$ pacing (middle panel) and biventricular (BV) pacing with VV delay of $80 \mathrm{~ms}$ (right panel). The total activation time, defined as time from onset of pacing until the end of the QRS complex, indicated between the two vertical dotted lines in each panel. During RV pacing, the total activation time is $218 \mathrm{~ms}$; however, it is increased during LV pacing until 274 ms. During BV pacing, this delayed activation can be compensated by preactivating LV $80 \mathrm{~ms}$ before RV

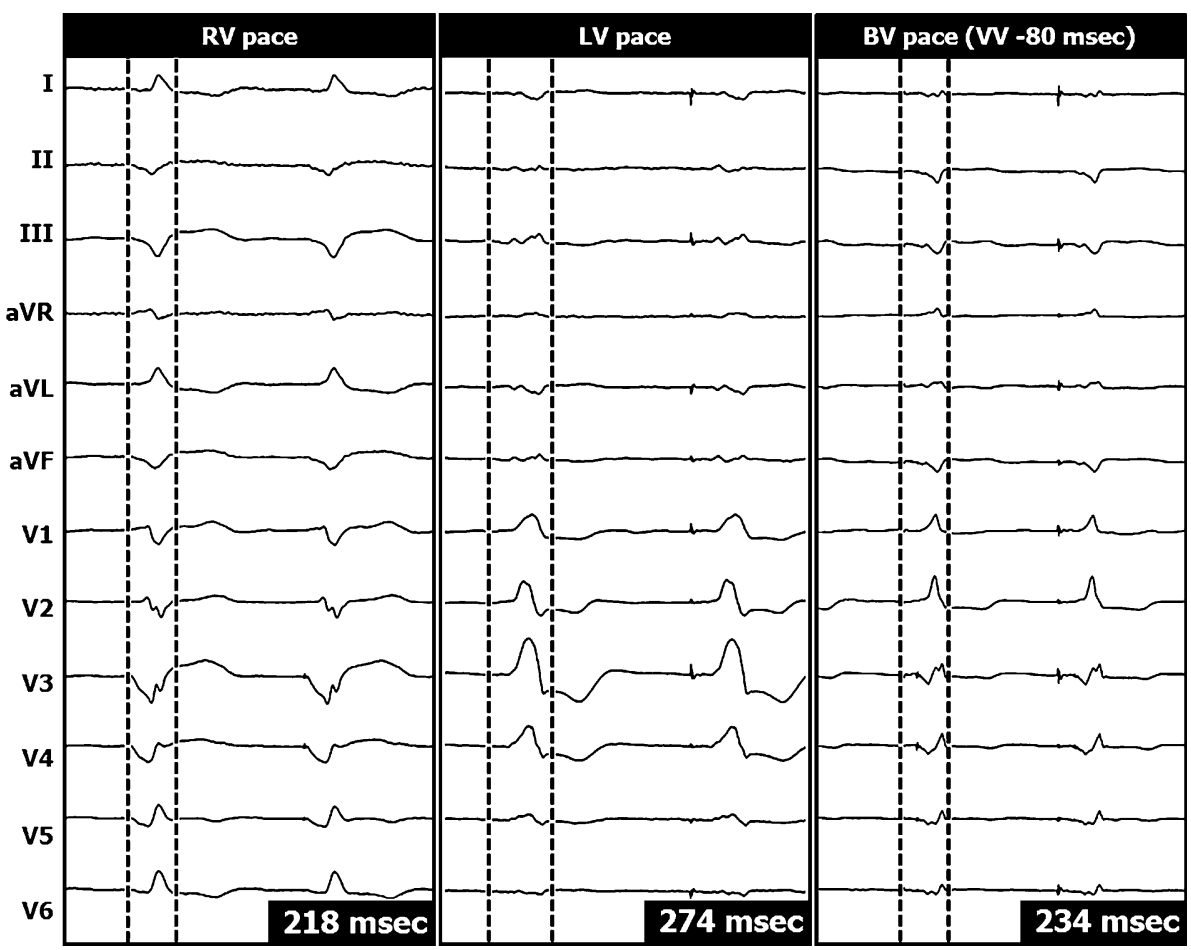

improvement in acute hemodynamic response measured by $\mathrm{LV} \mathrm{d} P / \mathrm{d} t_{\max }[39,40]$, exercise capacity [41] and echocardiographic left ventricular ejection fraction has been demonstrated [42, 43]. However, the larger, randomized
DECREASE-HF trial showed a trend toward greater reduction in left ventricular systolic diameter for the group with simultaneous biventricular pacing compared to sequential biventricular pacing [44], and the single- 
blinded, randomized RHYTHM-II trial did not find a benefit on functional endpoints of VV optimization compared to simultaneous biventricular pacing [45]. The recent randomized, multicenter RESPONSE-HF trial evaluated the effect of VV optimization on top of AV optimization. Patients who were non-responders after 3 months of CRT (with simultaneous biventricular pacing) were randomized to either sequential biventricular pacing with VV optimization or simultaneous biventricular pacing. Non-response was defined on the basis of NYHA class and 6-min hall walk distance. After 9 months of follow-up, the response rate in the sequential group $(n=29)$ was $18.9 \%$ higher than the simultaneous group $(n=36)$ [46].

In all but one of these studies [39], VV delay optimization was performed on top of prior AV optimization. In the overall CRT population, the benefit of VV optimization compared to simultaneous biventricular pacing is relatively small: van Gelder et al. noted a mean increase in LV $\mathrm{d} P / \mathrm{d} t_{\max }$ of $66 \mathrm{mmHg} / \mathrm{s}(7 \%)$ on top of simultaneous biventricular pacing with optimized AV delay [40]. VV optimization may probably be more beneficial in a subset of patients who show no or little response to CRT. It can be concluded on the basis of current data that the role of VV optimization is still under debate. This could partly be explained by the use of inaccurate optimization methods with high inter- and intraobserver variability.

There is no consensus in what order to optimize the AV and VV delays. However, in a small study, the hemodynamic effect (measured by fingerphotoplethysmography) of simultaneously adjusting AV and VV delays was evaluated. There was a curvilinear effect with a clear optimal combination of AV and VV delays. VV optimization provided an additional, but smaller hemodynamic effect compared to AV optimization alone [47].

\section{Intra-individual variation in optimal AV and VV delays}

The optimal AV and VV delays should not be regarded as static values, but may vary in time and in different circumstances. In general, optimization of AV and VV delays is performed during resting conditions in a supine or sitting position, thus neglecting the effect of exercise. In the healthy heart, AV conduction time shortens during exercise as a result of increased sympathetic tone and inter- and intraventricular activation delays are virtually absent and not different from the resting condition [48]. This is also the rationale for rate-adaptive atrial pacing with progressive shortening of the programmed AV delay during exercise. However, in the CRT population, it is questionable whether rate-adaptive pacing is favorable, as the effect of exercise on atrial and ventricular conduction is more heterogeneous and complex [49]. Several small studies investigating the effect of exercise on the optimal AV delay reported mixed results: some reported individual variation in optimal AV delay during exercise [50], others advise prolongation of the AV delay during exercise [51] while others notice no change in optimal AV delay [52].

VV optimization during exercise has been only sporadically investigated, using different optimization methods and including a limited number of patients. Lafitte et al. reported a change in interventricular dyssynchrony (defined as the interventricular mechanical delay) during bicycle exercise testing in $60 \%$ of 65 heart failure patients [48]. In contrast, Valzania et al. showed no significant change in interventricular mechanical delay during dobutamine stress testing [53]. Two other small studies showed that the optimal VV delay changes during bicycle exercise testing in about $55 \%$ of patients $[52,54]$. In one study in patients with atrial fibrillation and absent intrinsic AV conduction, a decrease in optimal VV delay with increasing pacing rate was noted [55].

Besides the effect of exercise, optimal AV and VV delays may also change in time as a result of reverse remodeling. Also here, data regarding the effect of longterm CRT on optimal AV and VV delays are limited and contradicting. In one study, there was a decrease in optimal AV delay and increase in LV pre-excitation in VV setting after 6 months of CRT [56]; however, another study showed an opposite effect after 9 months of CRT [57]. Although patient population was comparable, both trials used different optimization methods.

The large prospective, randomized and multicenter "Frequent Optimization Study Using the QuickOpt method (FREEDOM)" trial compared frequent AV and VV optimization every 3 months using an algorithm based on the intracardiac electrogram to standard care with empiric programming or one-time optimization at the discretion of the investigator. A heart failure clinical composite score was used as primary endpoint after 12 months of follow-up. In 1,525 patients analyzed, there was no significant difference in primary endpoint regardless of optimization [58].

\section{Methods for optimization of AV and VV delays}

There are numerous invasive and non-invasive methods available to optimize both AV and VV delays. It seems reasonable to assume that optimal delays result in highest forward stroke volume. The ideal optimization method should therefore be able to measure left ventricular (forward) stroke volume or an equivalent in a preferably reproducible, easy-to-perform and non-invasive way. 
Invasive optimization methods

\section{First derivate of left ventricular pressure pulse}

The ultimate way to determine contractile properties is measuring the force that is generated by a muscle; however, it not possible to measure this in clinical practice. As an alternative, the rate of left ventricular pressure change (LV dP/dt) has been proposed [59, 60]. Pressure is defined as force per unit area and is thus related to wall force. The rate of pressure development is influenced by the contractile properties of the LV. Changes in contractility alter the slope of the pressure curve resulting in an increased or decreased peak rise in intraventricular pressure $\left(\mathrm{d} P / \mathrm{d} t_{\max }\right)$ during isovolumetric contraction [61]. However, LV $\mathrm{d} P / \mathrm{d} t$ is a complex function that is not only dependent on contractility, but also dependent on preload, afterload and heart rate $[62,63]$. However, within physiological limits, $\mathrm{LV} \mathrm{d} P / \mathrm{d} t_{\max }$ shows mainly dependence on contractility and preload [64]. This properties make $\mathrm{LV} \mathrm{d} P / \mathrm{d} t_{\max }$ a useful instrument to evaluate the effect of both AV and VV delays on myocardial performance.

$\mathrm{LV} \mathrm{d} P / \mathrm{d} t$ is optimally derived from a left ventricular pressure curve obtained by a micromanometer that is introduced endovascular into the LV [65]. We have previously described an alternative method using a 0.014 highfidelity pressure wire (Radiwire, St. Jude Medical Inc., St. Paul, MN, USA) introduced either retrogradely or transseptally into the LV [40]. In order to adequately determine the effect of different pacing settings on LV $\mathrm{d} P / \mathrm{d} t_{\max }$, different protocols have been described $[65,66]$.

In order to overcome the influence of heart rate on LV $\mathrm{d} P / \mathrm{d} t$, the atrium is paced at $5-10$ beats above the intrinsic rate. In patients with atrial fibrillation, ventricular stimulation is performed above the intrinsic rate to ensure continuous capture. First, a baseline $\mathrm{LV} \mathrm{d} P / \mathrm{d} t_{\max }$ is measured and averaged out over several heart beats or seconds, excluding premature and post-extrasystolic beats from analysis. After baseline measurement, AV optimization is performed first during simultaneous biventricular pacing. The optimal AV delay with the highest $\mathrm{LV} \mathrm{d} P / \mathrm{d} t_{\max }$ is selected to perform the subsequent VV optimization. The optimization procedure should proceed under stable conditions to minimize any influence on $\mathrm{LV} \mathrm{d} P / \mathrm{d} t$ measurement.

This method has the advantage that it is easily implemented, even during the implantation procedure. Interpretation is not dependent on operator skills or technical limitations as with echocardiography. Also, it allows the evaluation of multiple pacing sites in a short time frame. Due to these characteristics, it is a suitable method to evaluate the acute hemodynamic effect of different pacing sites, either epicardially or even endocardially as has been demonstrated in a recent case report [67]. As an example, we implanted a left endocardial lead in a patient who showed no clinical or echocardiographic response to standard CRT. The definite LV pacing site was determined with optimal $\mathrm{LV} \mathrm{d} P / \mathrm{d} t_{\max }$ during a temporary pacing study of different endocardial sites. At long-term follow-up, there were both clinical and echocardiographic improvements.

A disadvantage of the $\mathrm{LV} \mathrm{d} P / \mathrm{d} t_{\max }$ optimization method is its invasive nature. However, as only a 4-French guiding is needed, no more complications than with standard angiography are to be expected. Nevertheless, in our opinion, the advantages of this invasive technique outweigh the relatively low risk. Alternatively, the pressure wire can be introduced via the radial artery or even via transseptal puncture.

The use of continuous wave Doppler imaging of the mitral regurgitation signal is advocated as a non-invasive alternative to determine $\mathrm{LV} \mathrm{d} P / \mathrm{d} t_{\max }$ [68]. Importantly, this method does not measure the true maximal $\mathrm{LV} \mathrm{d} P / \mathrm{d} t$, but an averaged slope of the left ventricular pressure curve between $4 \mathrm{mmHg}$ and $36 \mathrm{mmHg}$. This measure has not been validated in an experimental physiological set-up, as has been in case of invasively measured $\mathrm{LV} \mathrm{d} P / \mathrm{d} t_{\max }[61$, 63, 64]. Further, it requires the presence of a detectable mitral regurgitation signal which is not always present [69], has a lower temporal resolution than the invasive method and is more laborious to average over multiple heart beats.

Both PATH-CHF and PATH-CHF II trials used invasive $\mathrm{LV} \mathrm{d} P / \mathrm{d} t_{\max }$ to optimize the AV delay [30, 65]. So far, there are no randomized controlled trials evaluating the long-term outcome of CRT optimization by $\mathrm{LV} \mathrm{d} P / \mathrm{d} t_{\max }$.

\section{Pressure-volume loops}

LV pressure-volume loops can be used to calculate stroke work defined as the integrated area within the pressurevolume loop (in $\mathrm{mmHg} \mathrm{mL}$ ). This index is mainly dependent on contractility and preload with little effect of changes in afterload [64].

To acquire pressure-volume curves, a 6-French or 7-French pressure-conductance catheter is inserted in the LV via the femoral artery. The signals are digitized and transformed to pressure-volume loops by dedicated software $[64,70]$.

Except for its invasiveness, there are other disadvantages to the use of pressure-volume loops. The relatively inaccurate measurement of LV volume in dilated hearts combined with a low signal-to-noise ratio make it difficult to acquire a reliable signal in heart failure patients [71]. Also, the pressure-conductance catheter needs calibrating, has a larger size and is more expensive compared to the micromanometer used for left ventricular pressure measurements [72]. 
In contrary to $\mathrm{LV} \mathrm{d} P / \mathrm{d} t_{\max }$ measurement, the pressurevolume loop covers both the systolic and the diastolic phases of the cardiac cycle and incorporates both pressure and volume changes. This makes stroke work more sensitive to measure CRT-induced volume changes caused by alteration in mitral regurgitation. Further, the internal flow fraction derived from the conductance signals can be used to quantify LV mechanical dyssynchrony [73]. In selected cases, this dyssynchrony index could be used to support the indication for resynchronization therapy [74].

Compared to $\mathrm{LV} \mathrm{d} P / \mathrm{d} t_{\max }$, pressure-volume loops have been used only limited in early cardiac resynchronization studies [71]. Interestingly, when evaluating the acute hemodynamic response to CRT by both $\mathrm{LV} \mathrm{d} P / \mathrm{d} t_{\max }$ and stroke work, both measures do not match in up to $50 \%$ of the cases when using a cutoff value of $10 \%$ change to define response to CRT [72]. A sustained long-term hemodynamic response at 6 months has been demonstrated in a small-scale trial [70].

\section{Automated algorithms}

Several manufacturers of CRT devices have implemented automated algorithms to adjust AV and/or VV delays. As the optimal delays may change in time as a consequence of reverse remodeling after CRT as well as during exercise, these algorithms may be of additional value. However, adaptation during exercise can only be achieved if optimization performed continuously in a closed loop configuration. Optimization for reverse remodeling could be performed intermittently with automated algorithms.

\section{Algorithms based on the intracardiac electrogram}

QuickOpt. The QuickOpt algorithm (St. Jude Medical, St. Paul, MN, USA) has been designed to optimize both AV and VV delays using intracardiac electrograms. It has been demonstrated that the optimal AV delay can be calculated by measuring the time difference between onset of right atrial activation and end of left atrial activation using the intracardiac electrogram [75]. The QuickOpt algorithm uses the right intra-atrial electrogram to calculate the interatrial conduction delay. Depending on this delay, an offset is added to determine the optimal AV delay. For VV delay optimization, it is assumed that ventricular activation is optimal when the two depolarization wave fronts from right and left ventricular leads meet near the interventricular septum. The optimal VV delay is based on the conduction delay of both intrinsic rhythm and ventricular pacing. To measure this delay, intracardiac electrograms of both right and left ventricular leads are used. The interval between intrinsic activation of RV and LV leads is defined $\delta$, and the difference between RV pacing to LV sensing and
$\mathrm{LV}$ pacing to $\mathrm{RV}$ sensing is defined $\epsilon$. The optimal VV interval is then calculated using the formula $0.5(\delta+\epsilon)$. Although the algorithm shows a strong linear correlation with echocardiographic measurement of aortic velocity time integral [76], there is no correlation with the optimal $\mathrm{VV}$ delay determined by $\mathrm{LV} \mathrm{d} P / \mathrm{d} t_{\max }$ [77]. The correlation with the optimal AV and VV delays measured by echocardiography (using the iterative method for AV optimization and left ventricular outflow tract velocity time integral for VV optimization) is also poor [78].

The recent FREEDOM trial demonstrated that frequent optimization using QuickOpt did not significantly influence outcome as defined by the heart failure clinical composite score [58]. However, these results may be due to inaccuracy of the QuickOpt algorithm.

$S M A R T-A V$. The SMART-AV electrogram optimization algorithm (Boston Scientific Corporation, St. Paul, MN, USA) is part of the Expert Ease for Heart Failure feature and has been developed from results of large clinical trials [3, 30, 79]. Both sensed and paced AV delays are derived from the intracardiac electrogram and added to the QRS duration on the surface electrocardiogram in either mode. A correction factor is used depending on left ventricular lead position. This algorithm has been compared to two echocardiographic optimization methods (Ritter's and aortic velocity time integral method). In 28 patients examined, the electrogram optimization method correlated significantly better with LV $\mathrm{d} P / \mathrm{d} t_{\max }$ than the Ritter method [80]. The ongoing randomized, multicenter SMART-AV trial has been designed to compare the effect of different atrioventricular optimization methods on left ventricular remodeling. The electrogram optimization method will be compared to echocardiographic AV optimization (iterative method) and a fixed AV delay [81].

\section{Peak endocardial acceleration}

During the isovolumetric contraction period, the myocardium generates vibrations that are transmitted throughout the heart. The audible frequencies of these vibrations can be appreciated as the first (and second) heart sound. With a microaccelerometer (SonR, Sorin Biomedica, Saluggia, Italy) located on a lead inside the heart, it is possible to record the full-frequency spectrum and derive the peak endocardial acceleration (PEA). Early experimental research has shown that changes in PEA correlates well with changes in contractility induced by inotropic stimulation [82]. The optimal AV delay determined by PEA correlates well with those obtained by echocardiography (Ritter's method) [83-85]. In CRT, PEA increases significantly during $\mathrm{LV}$ or biventricular pacing compared to RV pacing only [86]. 
The randomized, multicenter CLinical Evaluation of Advanced Resynchronization (CLEAR) study compared $\mathrm{AV}$ and VV optimization by PEA to standard care for the composite endpoint of NYHA class, heart failure hospitalization and quality of life at 12 months in 186 patients. Patients optimized with PEA $(n=66)$ showed a significantly higher response rate [87].

\section{Finger photoplethysmography}

The conventional pulse oximetry probe measures the arterial pulsations of the fingertip vascular bed using a photo detector. It is possible to measure systolic blood pressure, pulse pressure and mean arterial pressure. As aortic pulse pressure is influenced by stroke volume and thus left ventricular performance, finger photoplethysmography may be used to optimize atrioventricular and interventricular delay. It seems a promising tool in cardiac resynchronization optimization because of its non-invasive nature and high reproducibility. However, measurements are highly influenced by waveform reflections in the arterial system and autonomic effects on peripheral resistance.

To overcome these issues, measurements are only taken a few beats after an atrioventricular delay change and an algorithm is used to correct for vasodilation and/or vasoconstriction. In patients who show a positive change in aortic pulse pressure during CRT (invasively measured), finger photoplethysmography (using the correction algorithm described) was able to predict the AV delay with the highest aortic pulse pressure change in up to $80 \%$ of the patients [88].

Another technique uses a volume-clamp circuit around the finger that dynamically follows arterial pressure (Finapres Medical System, Amsterdam, the Netherlands) [89]. The use of systolic blood pressure change measured by this technique responds to changing AV intervals and is claimed to be highly reproducible [89, 90]. Alternatively, Nexfin (BMEYE B.V., Amsterdam, the Netherlands) combines the volume-clamp technique with a dedicated algorithm to calculate stroke volume [91]. This method shows a good agreement with aortic valve velocity time integral to measure changes in stroke volume and to determine the optimal AV delay [92].

\section{Echocardiography}

Echocardiographic techniques for optimization of both AV and VV delays have been comprehensively described in recent review papers [27, 93, 94]. In general, echocardiography is a widely available and non-invasive technique without significant burden for the patient. However, these optimization techniques are subject of higher intra- and interobserver variability than invasive measurements. Still, echocardiography remains a cornerstone in CRT because of its ability to evaluate response to CRT in terms of reverse remodeling and to identify other factors that might influence a non-response to CRT (e.g. RV failure, pulmonary hypertension, valvular disease).

Evaluation of LV systolic function

Pulsed wave left ventricular outflow tract velocity time integral (LVOT-VTI). This parameter has been used to optimize both AV and VV delays. In a few small-scale, uncontrolled studies, the optimal AV delay was defined as the delay with the highest stroke distance measured by LVOT-VTI, but there is no correlation with outcome [35, 51]. In a post hoc analysis, the InSync III study compared VV optimization using LVOT stroke volume to simultaneous biventricular pacing. There was only a significant improvement in 6-min walking test (6MWT) compared to the control group; quality of life and NYHA class were not significantly different [95]. Also, the previously described RHYTHM II ICD trial used LVOT-VTI measurements for VV optimization, but reported no benefit on functional endpoints [45]. One small, non-randomized study used LVOT-VTI to optimize both AV and VV delays after 3 months of non-optimized CRT and concluded that the method was feasible, reproducible and able to improve response to CRT [96].

Continuous wave aortic valve velocity time integral (AV-VTI). Sawhney et al. showed in a randomized, prospective trial in 40 patients that compared to an empirical AV delay of $120 \mathrm{~ms}$, AV optimization using AV-VTI yields a significant improvement in NYHA class, quality of life and 6MWT [33]. Another prospective study in 40 patients compared AV optimization by AV-VTI to the Ritter's method and concluded that the AV-VTI method resulted in greater systolic improvement [97]. However, the methodology of both studies has been questioned [27].

$L V d P / d t$. Even though proposed as a surrogate for invasive $\mathrm{LV} \mathrm{d} P / \mathrm{d} t_{\max }$ measurement [68], this measurement is not recommended as optimization method as reproducibility has been reported as suboptimal [96].

Tissue Doppler imaging (TDI). Although TDI has the potential to assess left ventricular dyssynchrony, it is subject to high inter- and intra-observer variability [96, 98]. TDI was used to optimize VV delay and was compared to empirical AV and VV delays by Vidal et al. in 100 patients [99]. The optimal VV delay was defined as the setting with the greatest superposition of TDI curves of opposing LV walls in 2-chamber and 4-chamber view. There was only a significant improvement in 6MWT in the optimized group. However, $25 \%$ of patients in the optimized group did not receive $\mathrm{AV}$ optimization because of atrial fibrillation and a power calculation justifying the included number of 
patients is lacking. Another study used TDI based on measurement of regional electromechanical delay of $18 \mathrm{LV}$ segments in the 3 apical views. The AV and VV delays were defined as optimal when the basal septal segment and the segments containing the right and left ventricular leads (as identified by computer tomography) were synchronized. Comparing a limited number of VV intervals, derived optimal VV delay coincided with the greatest cardiac output as measured by thermodilution. Although complex and time-consuming, this method is one of the few based on the underlying physiological concept of synchronizing the three activation fronts [100].

\section{Evaluation of LV diastolic function}

Iterative method. The AV delay is shortened by increments of $20 \mathrm{~ms}$ until truncation of the A-wave on the pulsed Doppler transmitral flow pattern. Next, the AV delay is increased again by increments of $10 \mathrm{~ms}$ until A-wave truncation disappears. The latter is defined as the optimal AV delay. The iterative method was used for AV optimization in the CARE-HF trial and the aforementioned study of Vidal et al. [99, 101].

Ritter's formula. This method was originally proposed for patients with complete heart block [102]. Even though it has only been presented as an abstract and no further validation has been published, its use has been extrapolated to the CRT population without extensive validation. The formula defines the optimal AV delay as the AV interval that bridges the end of the A-wave with closure of the mitral valve or the onset of ventricular contraction. To do so, the time from onset of QRS complex to time of termination of the A-wave (QA interval) is measured at both a long $\left(\mathrm{AV}_{\text {long }}\right)$ and a short $\mathrm{AV}$ delays $\left(\mathrm{AV}_{\text {short }}\right)$. The optimal AV delay is calculated from following formula: $\mathrm{AV}_{\text {opt }}=\mathrm{AV}_{\text {long }}-\left(\mathrm{QA}_{\text {short }}-\mathrm{QA}_{\text {long }}\right)$. Ritter's formula has also been compared to the QuickOpt algorithm and $\mathrm{AV}-\mathrm{VTI}$ for $\mathrm{AV}$ optimization using $\mathrm{LV} \mathrm{d} P / \mathrm{d} t_{\max }$ as gold standard. This study showed that Ritter's formula was least accurate [80].

Mitral inflow velocity time integral. On the pulsed Doppler transmitral flow pattern, the VTI is calculated representing the stroke distance of mitral inflow as a surrogate of LV filling volume. The AV delay with the largest VTI is considered the optimal setting. The method showed a good correlation with optimization by $\mathrm{LV} \mathrm{d} P / \mathrm{d} t_{\max }$ ( $r=0.96)$ in a small study of 30 patients [103].

Meluzin's method. A simplified method to merge the end of atrial contraction with mitral valve closure was proposed by Meluzin [104]. A long AV delay is programmed and the pulsed Doppler transmitral inflow pattern is recorded. The time between end of the A-wave and onset of systolic mitral regurgitation is calculated. This time is subtracted from the programmed AV delay to determine the optimal AV interval. The method was only validated in a study of 18 patients which showed a significantly higher cardiac output measured by thermodilution when comparing the optimal AV delay to longer and shorter AV delays [104]. Obviously, application of this method is dependent on a clear mitral regurgitation signal.

Evaluation of LV systolic and diastolic function

Myocardial performance index (MPI). The MPI (or Tei index) is based on cardiac timing intervals and has been introduced as a measurement incorporating both LV systolic and diastolic functions. The mitral-closure-to-opening (MCO) interval is measured on the pulsed Doppler transmitral flow signal, and the ejection time (ET) is derived from the pulsed Doppler LVOT flow signal. As the total of the isovolumetric contraction and relaxation time (ICT and IRT) is obtained by subtracting ET from MCO, the index incorporates both systolic and diastolic indices [105]. Two small studies used MPI to optimize AV delay [106, 107] and/or VV delay [106]. Both studies lacked a control group and well-defined endpoints.

\section{Conclusion}

Experimental physiological and pathophysiological research supports the rationale to optimize AV and VV delays in CRT. Although there is a spectrum of possible optimization methods, no evident golden standard has emerged, partly due to the lack of large-scale studies evaluating these methods to outcome. Thus, at present, no single method can be recommended for standard practice. Present studies support the physiological rationale for AV optimization, but data concerning VV optimization are still conflicting. As the incremental benefit of VV optimization is relatively small, the effect is probably more of importance in a subset of CRT patients (with special attention for non-responders). Although current efforts mainly investigate optimization during resting conditions, there is a need to develop automated algorithms to implement dynamic optimization in order to adapt to physiological alterations during exercise and after anatomical remodeling.

Conflict of interest Berry M. van Gelder is a consultant for Medtronic Trading NL B.V. All other authors state that they have no conflicting interests to declare.

Open Access This article is distributed under the terms of the Creative Commons Attribution Noncommercial License which permits any noncommercial use, distribution, and reproduction in any medium, provided the original author(s) and source are credited. 


\section{References}

1. Bristow MR, Saxon LA, Boehmer J et al (2004) Cardiac resynchronization therapy with or without an implantable defibrillator in advanced chronic heart failure. N Engl J Med 350(21):2140-2150

2. Cleland JG, Daubert JC, Erdmann E et al (2005) The effect of cardiac resynchronization on morbidity and mortality in heart failure. N Engl J Med 352(15):1539-1549

3. Abraham WT, Fisher WG, Smith AL et al (2002) Cardiac resynchronization in chronic heart failure. $N$ Engl $\mathrm{J}$ Med 346(24):1845-1853

4. Durrer D, van Dam RT, Freud GE, Janse MJ, Meijler FL, Arzbaecher RC (1970) Total excitation of the isolated human heart. Circulation 41(6):899-912

5. Prinzen FW, Spinelli J, Auricchio A (2007) Basic physiology and hemodynamics of cardiac pacing. In: Ellenbogen KA, Kay GN, Lau CP, Wilkoff B (eds) Clinical cardiac pacing, defibrillation, and resynchronization therapy. Saunders Elsevier, Philadelphia, pp 291-335

6. Mitchell J, Gilmore J, Sarnoff S (1962) The transport function of the atrium. Factors influencing the relation between mean left atrial pressure and left ventricular end diastolic pressure. Am J Cardiol 9:237-247

7. Burchell H (1964) A clinical appraisal of atrial transport function. Lancet 1(7337):775-779

8. Skinner N Jr, Mitchell J, Wallace A, Sarnoff S (1963) Hemodynamic effects of altering the timing of atrial systole. Am J Physiol 205:499-503

9. Fung JW, Yu CM, Yip G et al (2004) Variable left ventricular activation pattern in patients with heart failure and left bundle branch block. Heart 90(1):17-19

10. Frazier DW, Krassowska W, Chen PS et al (1988) Transmural activations and stimulus potentials in three-dimensional anisotropic canine myocardium. Circ Res 63(1):135-146

11. Weber KT, Janicki JS, Shroff S, Fishman AP (1981) Contractile mechanics and interaction of the right and left ventricles. Am J Cardiol 47(3):686-695

12. Antoni H (1996) Functional properties of the heart. In: Greger R, Windhorst U (eds) Comprehensive human physiology. From cellular mechanisms to integration, vol 2. Springer Verlag, Berlin, pp 1801-1824

13. Farrar DJ, Woodard JC, Chow E (1993) Pacing-induced dilated cardiomyopathy increases left-to-right ventricular systolic interaction. Circulation 88(2):720-725

14. Atherton JJ, Moore TD, Lele SS et al (1997) Diastolic ventricular interaction in chronic heart failure. Lancet 349(9067): $1720-1724$

15. Kosowsky BD, Scherlag BJ, Damato AN (1968) Re-evaluation of the atrial contribution to ventricular function: study using His bundle pacing. Am J Cardiol 21(4):518-524

16. Vernooy K, Verbeek XA, Cornelussen RN et al (2007) Calculation of effective $\mathrm{VV}$ interval facilitates optimization of $\mathrm{AV}$ delay and $\mathrm{VV}$ interval in cardiac resynchronization therapy. Heart Rhythm 4(1):75-82

17. Prinzen FW, Hunter WC, Wyman BT, McVeigh ER (1999) Mapping of regional myocardial strain and work during ventricular pacing: experimental study using magnetic resonance imaging tagging. J Am Coll Cardiol 33(6):1735-1742

18. Cazeau S, Leclercq C, Lavergne T et al (2001) Effects of multisite biventricular pacing in patients with heart failure and intraventricular conduction delay. N Engl J Med 344(12):873-880

19. Samet P, Castillo C, Bernsein WH (1968) Hemodynamic consequences of sequential atrioventricular pacing. Subjects with normal hearts. Am J Cardiol 21(2):207-212
20. Wilkoff BL, Cook JR, Epstein AE et al (2002) Dual-chamber pacing or ventricular backup pacing in patients with an implantable defibrillator: the Dual Chamber and VVI Implantable Defibrillator (DAVID) trial. JAMA 288(24): 3115-3123

21. Hochleitner M, Hortnagl H, Ng CK, Hortnagl H, Gschnitzer F, Zechmann W (1990) Usefulness of physiologic dual-chamber pacing in drug-resistant idiopathic dilated cardiomyopathy. Am J Cardiol 66(2):198-202

22. Gold MR, Feliciano Z, Gottlieb SS, Fisher ML (1995) Dualchamber pacing with a short atrioventricular delay in congestive heart failure: a randomized study. J Am Coll Cardiol 26(4):967-973

23. Nishimura RA, Hayes DL, Holmes DR Jr, Tajik AJ (1995) Mechanism of hemodynamic improvement by dual-chamber pacing for severe left ventricular dysfunction: an acute Doppler and catheterization hemodynamic study. J Am Coll Cardiol 25(2):281-288

24. Breithardt OA, Sinha AM, Schwammenthal E et al (2003) Acute effects of cardiac resynchronization therapy on functional mitral regurgitation in advanced systolic heart failure. J Am Coll Cardiol 41(5):765-770

25. Kanzaki H, Bazaz R, Schwartzman D, Dohi K, Sade LE, Gorcsan J III (2004) A mechanism for immediate reduction in mitral regurgitation after cardiac resynchronization therapy: insights from mechanical activation strain mapping. J Am Coll Cardiol 44(8):1619-1625

26. Ypenburg C, Lancellotti P, Tops LF et al (2008) Mechanism of improvement in mitral regurgitation after cardiac resynchronization therapy. Eur Heart J 29(6):757-765

27. Stanton T, Hawkins NM, Hogg KJ, Goodfield NE, Petrie MC, McMurray JJ (2008) How should we optimize cardiac resynchronization therapy? Eur Heart J 29(20):2458-2472

28. Auricchio A, Stellbrink C, Block M et al (1999) Effect of pacing chamber and atrioventricular delay on acute systolic function of paced patients with congestive heart failure. The Pacing Therapies for Congestive Heart Failure Study Group. The Guidant Congestive Heart Failure Research Group. Circulation 99(23): 2993-3001

29. Auricchio A, Ding J, Spinelli JC et al (2002) Cardiac resynchronization therapy restores optimal atrioventricular mechanical timing in heart failure patients with ventricular conduction delay. J Am Coll Cardiol 39(7):1163-1169

30. Butter C, Auricchio A, Stellbrink C et al (2001) Effect of resynchronization therapy stimulation site on the systolic function of heart failure patients. Circulation 104(25):3026-3029

31. van Gelder BM, Bracke FA, Meijer A, Pijls NH (2005) The hemodynamic effect of intrinsic conduction during left ventricular pacing as compared to biventricular pacing. J Am Coll Cardiol 46(12):2305-2310

32. Van Gelder BM, Bracke FA, VoP Van Der, Meijer A (2007) Optimal sensed atrio-ventricular interval determined by paced QRS morphology. Pacing Clin Electrophysiol 30(4):476-481

33. Sawhney NS, Waggoner AD, Garhwal S, Chawla MK, Osborn J, Faddis MN (2004) Randomized prospective trial of atrioventricular delay programming for cardiac resynchronization therapy. Heart Rhythm 1(5):562-567

34. Morales MA, Startari U, Panchetti L, Rossi A, Piacenti M (2006) Atrioventricular delay optimization by doppler-derived left ventricular $\mathrm{dP} / \mathrm{dt}$ improves 6-month outcome of resynchronized patients. Pacing Clin Electrophysiol 29(6):564-568

35. Hardt SE, Yazdi SH, Bauer A et al (2007) Immediate and chronic effects of AV-delay optimization in patients with cardiac resynchronization therapy. Int J Cardiol 115(3):318325 
36. Vardas PE, Auricchio A, Blanc JJ et al (2007) Guidelines for cardiac pacing and cardiac resynchronization therapy: the task force for cardiac pacing and cardiac resynchronization therapy of the European society of cardiology. Developed in collaboration with the European Heart Rhythm Association. Eur Heart J 28(18):2256-2295

37. Asirvatham SJ, Hayes DL (2004) Optimization of biventricular devices. In: Hayes DL, Wang P, Sackner-Bernstein J, Asirvatham SJ (eds) Resynchronization and defibrillation for heart failure. A practical approach. Blackwell Publishing, Oxford, pp 139-162

38. Mortensen PT, Sogaard P, Mansour H et al (2004) Sequential biventricular pacing: evaluation of safety and efficacy. Pacing Clin Electrophysiol 27(3):339-345

39. Perego GB, Chianca R, Facchini M et al (2003) Simultaneous vs. sequential biventricular pacing in dilated cardiomyopathy: an acute hemodynamic study. Eur J Heart Fail 5(3):305-313

40. van Gelder BM, Bracke FA, Meijer A, Lakerveld LJ, Pijls NH (2004) Effect of optimizing the VV interval on left ventricular contractility in cardiac resynchronization therapy. Am J Cardiol 93(12):1500-1503

41. Leon AR, Abraham WT, Brozena S et al (2005) Cardiac resynchronization with sequential biventricular pacing for the treatment of moderate-to-severe heart failure. J Am Coll Cardiol 46(12):2298-2304

42. Sogaard P, Egeblad H, Pedersen AK et al (2002) Sequential versus simultaneous biventricular resynchronization for severe heart failure: evaluation by tissue Doppler imaging. Circulation 106(16):2078-2084

43. Vanderheyden M, De Backer T, Rivero-Ayerza M et al (2005) Tailored echocardiographic interventricular delay programming further optimizes left ventricular performance after cardiac resynchronization therapy. Heart Rhythm 2(10):1066-1072

44. Rao RK, Kumar UN, Schafer J, Viloria E, De Lurgio D, Foster E (2007) Reduced ventricular volumes and improved systolic function with cardiac resynchronization therapy: a randomized trial comparing simultaneous biventricular pacing, sequential biventricular pacing, and left ventricular pacing. Circulation 115(16):2136-2144

45. Boriani G, Muller CP, Seidl KH et al (2006) Randomized comparison of simultaneous biventricular stimulation versus optimized interventricular delay in cardiac resynchronization therapy. The Resynchronization for the HemodYnamic Treatment for Heart Failure Management II implantable cardioverter. Am Heart J 151(5):1050-1058

46. Weiss R, Malik M, Dinerman J, Lee L, Petrutiu S, Khoo M (2010) V-V optimization in cardiac resynchronization therapy non-responders: RESPONSE-HF trail results. Heart Rhythm 7(5):S26

47. Whinnett ZI, Davies JE, Willson K et al (2006) Haemodynamic effects of changes in atrioventricular and interventricular delay in cardiac resynchronisation therapy show a consistent pattern: analysis of shape, magnitude and relative importance of atrioventricular and interventricular delay. Heart 92(11):1628-1634

48. Lafitte S, Bordachar P, Lafitte M et al (2006) Dynamic ventricular dyssynchrony: an exercise-echocardiography study. J Am Coll Cardiol 47(11):2253-2259

49. Bogaard MD, Kirkels JH, Hauer RN, Loh P, Doevendans PA, Meine M (2010) Should we optimize cardiac resynchronization therapy during exercise? J Cardiovasc Electrophysiol 21(11): $1307-1316$

50. Mokrani B, Lafitte S, Deplagne A et al (2009) Echocardiographic study of the optimal atrioventricular delay at rest and during exercise in recipients of cardiac resynchronization therapy systems. Heart Rhythm 6(7):972-977
51. Scharf C, Li P, Muntwyler J et al (2005) Rate-dependent AV delay optimization in cardiac resynchronization therapy. Pacing Clin Electrophysiol 28(4):279-284

52. Valzania C, Eriksson MJ, Boriani G, Gadler F (2008) Cardiac resynchronization therapy during rest and exercise: comparison of two optimization methods. Europace 10(10):1161-1169

53. Valzania C, Gadler F, Eriksson MJ, Olsson A, Boriani G, Braunschweig F (2007) Electromechanical effects of cardiac resynchronization therapy during rest and stress in patients with heart failure. Eur J Heart Fail 9(6-7):644-650

54. Bordachar P, Lafitte S, Reuter S et al (2006) Echocardiographic assessment during exercise of heart failure patients with cardiac resynchronization therapy. Am J Cardiol 97(11):1622-1625

55. van Gelder BM, Meijer A, Bracke FA (2008) Stimulation rate and the optimal interventricular interval during cardiac resynchronization therapy in patients with chronic atrial fibrillation. Pacing Clin Electrophysiol 31(5):569-574

56. Porciani MC, Dondina C, Macioce R et al (2006) Temporal variation in optimal atrioventricular and interventricular delay during cardiac resynchronization therapy. J Card Fail 12(9): 715-719

57. O'Donnell D, Nadurata V, Hamer A, Kertes P, Mohamed U (2005) Long-term variations in optimal programming of cardiac resynchronization therapy devices. Pacing Clin Electrophysiol 28(Suppl 1):S24-S26

58. Abraham WT, Gras D, Yu CM et al (2010) Results from the freedom trial-assess the safety and efficacy of frequent optimization of cardiac resynchronization therapy. Heart Rhythm 7(5):2-3

59. Mason DT, Braunwald E, Covell JW, Sonnenblick EH, Ross J Jr (1971) Assessment of cardiac contractility. The relation between the rate of pressure rise and ventricular pressure during isovolumic systole. Circulation 44(1):47-58

60. van den Bos GC (1972) Indices of contractility in the intact heart. Proc R Soc Med 65(6):545-547

61. Reeves T, Hefner L, Jones W et al. (1960) The hemodynamic determinants of the rate of change in pressure in the left ventricle during isometric contraction. Am Heart J 60:745-761

62. Wiggers CJ (1914) Some factors controlling the shape of the pressure curve in the right ventricle. Am J Physiol 33(3): 382-396

63. Wallace A, Skinner N Jr, Mitchell J (1963) Hemodynamic determinants of the maximal rate of rise of left ventricular pressure. Am J Physiol 205:30-36

64. Kass DA, Maughan WL, Guo ZM, Kono A, Sunagawa K, Sagawa K (1987) Comparative influence of load versus inotropic states on indexes of ventricular contractility: experimental and theoretical analysis based on pressure-volume relationships. Circulation 76(6):1422-1436

65. Auricchio A, Stellbrink C, Sack S et al (1999) The pacing therapies for congestive heart failure (PATH-CHF) study: rationale, design, and endpoints of a prospective randomized multicenter study. Am J Cardiol 83(5B):130D-135D

66. Jansen AH, van Gelder BM (2008) Visual LV motion and invasive $\mathrm{LVdP} / \mathrm{dtmax}$ for selection and optimisation of cardiac resynchronisation therapy. Neth Heart J 16(Suppl 1):S32S35

67. Bracke FA, Houthuizen P, Rahel BM, van Gelder BM (2010) Left ventricular endocardial pacing improves the clinical efficacy in a non-responder to cardiac resynchronization therapy: role of acute haemodynamic testing. Europace 12(7):1032-1034

68. Bargiggia GS, Bertucci C, Recusani F et al (1989) A new method for estimating left ventricular $\mathrm{dP} / \mathrm{dt}$ by continuous wave Doppler-echocardiography. Validation studies at cardiac catheterization. Circulation 80(5):1287-1292 
69. Tournoux FB, Alabiad C, Fan D et al (2007) Echocardiographic measures of acute haemodynamic response after cardiac resynchronization therapy predict long-term clinical outcome. Eur Heart J 28(9):1143-1148

70. Steendijk P, Tulner SA, Bax JJ et al (2006) Hemodynamic effects of long-term cardiac resynchronization therapy: analysis by pressure-volume loops. Circulation 113(10):1295-1304

71. Kass DA, Chen CH, Curry C et al (1999) Improved left ventricular mechanics from acute VDD pacing in patients with dilated cardiomyopathy and ventricular conduction delay. Circulation 99(12): 1567-1573

72. de Roest G, Knaapen P, Gotte M et al (2009) Stroke work or systolic $\mathrm{dP} / \mathrm{dtmax}$ to evaluate acute response to cardiac resynchronization therapy: are they interchangeable? Eur J Heart Fail 11(7):706-708

73. Steendijk P, Tulner SA, Schreuder JJ et al (2004) Quantification of left ventricular mechanical dyssynchrony by conductance catheter in heart failure patients. Am J Physiol Heart Circ Physiol 286(2):H723-H730

74. Penicka M, Kocka V, Herman D, Trakalova H, Herold M (2010) Cardiac resynchronization therapy for the causal treatment of heart failure with preserved ejection fraction: insight from a pressure-volume loop analysis. Eur J Heart Fail 55(16):17011710

75. Worley SJ, Gohn C, Smith T (2004) Optimization of cardiac resynchronization: left atrial electrograms measured at implant eliminates the need for echo and identifies patients where AV optimization is not possible. J Card Fail 10(4):S62

76. Baker J II, McKenzie J III, Beau S et al (2007) Acute evaluation of programmer-guided AV/PV and VV delay optimization comparing an IEGM method and echocardiogram for cardiac resynchronization therapy in heart failure patients and dualchamber ICD implants. J Cardiovasc Electrophysiol 18(2): 185-191

77. van Gelder BM, Meijer A, Bracke FA (2008) The optimized $\mathrm{V}-\mathrm{V}$ interval determined by interventricular conduction times versus invasive measurement by LVdP/dtMAX. J Cardiovasc Electrophysiol 19(9):939-944

78. Kamdar R, Frain E, Warburton F et al (2010) A prospective comparison of echocardiography and device algorithms for atrioventricular and interventricular interval optimization in cardiac resynchronization therapy. Europace 12(1):84-91

79. Auricchio A, Stellbrink C, Sack S et al (2002) Long-term clinical effect of hemodynamically optimized cardiac resynchronization therapy in patients with heart failure and ventricular conduction delay. J Am Coll Cardiol 39(12):2026-2033

80. Gold MR, Niazi I, Giudici M et al (2007) A prospective comparison of AV delay programming methods for hemodynamic optimization during cardiac resynchronization therapy. J Cardiovasc Electrophysiol 18(5):490-496

81. Corporation BS (2008) Comparison of AV optimization methods used in cardiac resynchronization therapy (CRT) (SMARTAV). http://www.clinicaltrials.gov/ct2/show/NCT00677014

82. Rickards AF, Bombardini T, Corbucci G, Plicchi G (1996) An implantable intracardiac accelerometer for monitoring myocardial contractility. The multicenter PEA study group. Pacing Clin Electrophysiol 19(12 Pt 1):2066-2071

83. Ritter P, Padeletti L, Gillio-Meina L, Gaggini G (1999) Determination of the optimal atrioventricular delay in DDD pacing. Comparison between echo and peak endocardial acceleration measurements. Europace 1(2):126-130

84. Leung SK, Lau CP, Lam CT et al (2000) Automatic optimization of resting and exercise atrioventricular interval using a peak endocardial acceleration sensor: validation with Doppler echocardiography and direct cardiac output measurements. Pacing Clin Electrophysiol 23(11 Pt 2):1762-1766
85. Dupuis JM, Kobeissi A, Vitali L et al (2003) Programming optimal atrioventricular delay in dual chamber pacing using peak endocardial acceleration: comparison with a standard echocardiographic procedure. Pacing Clin Electrophysiol 26(1 Pt 2):210-213

86. Bordachar P, Garrigue S, Reuter S et al (2000) Hemodynamic assessment of right, left, and biventricular pacing by peak endocardial acceleration and echocardiography in patients with end-stage heart failure. Pacing Clin Electrophysiol 23(11 Pt 2): $1726-1730$

87. Ritter P, Nagele H, Lunati M et al (2010) Clinical benefit of cardiac resynchronization therapy patients optimized by SonR or standard methods: final results from the CLEAR study. Europace 12(Suppl 1):i50

88. Butter C, Stellbrink C, Belalcazar A et al (2004) Cardiac resynchronization therapy optimization by finger plethysmography. Heart Rhythm 1(5):568-575

89. Whinnett ZI, Davies JE, Willson K et al (2006) Determination of optimal atrioventricular delay for cardiac resynchronization therapy using acute non-invasive blood pressure. Europace $8(5): 358-366$

90. Whinnett ZI, Davies JE, Nott G et al (2008) Efficiency, reproducibility and agreement of five different hemodynamic measures for optimization of cardiac resynchronization therapy. Int $\mathbf{J}$ Cardiol 129(2):216-226

91. Eeftinck Schattenkerk D, van Lieshout J, van den Meiracker A et al (2009) Nexfin noninvasive continuous blood pressure validation against Riva-Rocci/Korotkoff. Am J Hypertens 22(4): 378-383

92. van Geldorp IE, Delhaas T, Hermans B et al. (2010) Comparison of a non-invasive arterial pulse contour technique and echo Doppler aorta velocity-time integral on stroke volume changes in optimization of cardiac resynchronization therapy. Europace. doi:10.1093/europace/euq348

93. Bhan A, Kapetanakis S, Monaghan MJ (2008) Optimization of cardiac resynchronization therapy. Echocardiography 25(9): 1031-1039

94. Bertini M, Delgado V, Bax JJ, Van de VN (2009) Why, how and when do we need to optimize the setting of cardiac resynchronization therapy? Europace 11(Suppl 5):v46-v57

95. Leon AR, Abraham WT, Curtis AB et al (2005) Safety of transvenous cardiac resynchronization system implantation in patients with chronic heart failure: combined results of over 2, 000 patients from a multicenter study program. J Am Coll Cardiol 46(12):2348-2356

96. Thomas DE, Yousef ZR, Fraser AG (2009) A critical comparison of echocardiographic measurements used for optimizing cardiac resynchronization therapy: stroke distance is best. Eur J Heart Fail 11(8):779-788

97. Kerlan JE, Sawhney NS, Waggoner AD et al (2006) Prospective comparison of echocardiographic atrioventricular delay optimization methods for cardiac resynchronization therapy. Heart Rhythm 3(2): 148-154

98. Chung ES, Leon AR, Tavazzi L et al (2008) Results of the predictors of response to CRT (PROSPECT) trial. Circulation 117(20):2608-2616

99. Vidal B, Sitges M, Marigliano A et al (2007) Optimizing the programation of cardiac resynchronization therapy devices in patients with heart failure and left bundle branch block. Am J Cardiol 100(6):1002-1006

100. Novak M, Lipoldova J, Meluzin J et al (2008) Contribution to the V-V interval optimization in patients with cardiac resynchronization therapy. Physiol Res 57(5):693-700

101. Cleland JG, Daubert JC, Erdmann E et al (2001) The CARE-HF study (CArdiac REsynchronisation in Heart Failure study): rationale, design and end-points. Eur J Heart Fail 3(4):481-489 
102. Ritter P, Lelieve T, Lavergne T (1994) Quick determination of the optimal AV delay at rest in patients paced in DDD mode for complete AV block. Eur J Cardiac Pacing Electrophysiol 4(2):A163

103. Jansen AH, Bracke FA, van Dantzig JM et al (2006) Correlation of echo-Doppler optimization of atrioventricular delay in cardiac resynchronization therapy with invasive hemodynamics in patients with heart failure secondary to ischemic or idiopathic dilated cardiomyopathy. Am J Cardiol 97(4): 552-557

104. Meluzin J, Novak M, Mullerova J et al (2004) A fast and simple echocardiographic method of determination of the optimal atrioventricular delay in patients after biventricular stimulation. Pacing Clin Electrophysiol 27(1):58-64
105. Tei C, Ling LH, Hodge DO et al (1995) New index of combined systolic and diastolic myocardial performance: a simple and reproducible measure of cardiac function-a study in normals and dilated cardiomyopathy. J Cardiol 26(6):357-366

106. Porciani MC, Dondina C, Macioce R et al (2005) Echocardiographic examination of atrioventricular and interventricular delay optimization in cardiac resynchronization therapy. Am J Cardiol 95(9): 1108-1110

107. Stockburger M, Fateh-Moghadam S, Nitardy A, Langreck H, Haverkamp W, Dietz R (2006) Optimization of cardiac resynchronization guided by Doppler echocardiography: haemodynamic improvement and intraindividual variability with different pacing configurations and atrioventricular delays. Europace $8(10): 881-886$ 